\title{
On Separation Axioms in Fuzzifying Generalized Topology
}

\author{
N. Gowrisankar, N. Rajesh and V. Vijayabharathi
}

Abstract. In this paper we introduce and study the concept of fuzzifying separation axioms in fuzzifying generalized topological spaces.

\section{INTRODUCTION}

Fuzzy topology as an important research field in fuzzy set theory has been developed into quite a mature discipline [1]-[7]. In contrast to classical topology, fuzzy topology is endowed with richer structure to a certain extent, which is manifested in different ways to generalize certain classical concepts. So far, according to [2], the kind of topologies defined by Chang [8] and Goguen [9] are called the topologies of fuzzy subsets, and further are naturally called $L$-topological spaces if a lattice $L$ of membership values has been chosen. Loosely speaking, a topology of fuzzy subsets (resp. an $L$-topological space) is a family $\mu$ of fuzzy subsets (resp. $L$-fuzzy subsets) of nonempty set $X$, and $\mu$ satisfies the basic conditions of classical topologies. The concept of fuzzifying generalized topology was introduced and studied by the same authors [10]. All the results in this paper as a generalization of the results in [4], [11], [12] and [13]. That is, we introduce and study the concept of fuzzifying separation axioms in fuzzifying generalized topological spaces.

\section{Preliminaries}

First, we display the Lukasiewicz logic and corresponding set theoretical notations used in this paper. For any formula $\varphi$, the symbol $[\varphi]$ means the truth value of $\varphi$, where the set of truth values is the unit interval $[0,1]$. We write $=\varphi$ if $[\varphi]=1$ for any interpretation. By $=^{w} \varphi(\varphi$ is feebly valid $)$ we mean that for any valuation it always holds that $[\varphi]>0$, and $\varphi \models$ ws $\xi$

2010 Mathematics Subject Classification. Primary: 54A40.

Key words and phrases. Lukasiewicz logic, generalized, fuzzifying Generalized topology, fuzzifying $\mu$-open sets. 
we mean that $[\varphi]>0$ implies $[\xi]=1$. The truth valuation rules for primary fuzzy logical formulae and corresponding set theoretical notations are:

(1) (a) $[\lambda]=\lambda(\lambda \in[0,1])$;

(b) $[\varphi \wedge \xi]=\min ([\varphi],[\xi])$;

(c) $[\varphi \rightarrow \xi]=\min (1,1-[\varphi]+[\xi])$;

(2) If $\tilde{A} \in \Im(X)$, then $[x \in \tilde{A}]:=\tilde{A}(x)$.

(3) If $X$ is the universe of discourse, then $[\forall x \varphi(x)]:=\inf _{x \in X}[\varphi(x)]$.

In addition, the truth valuation rules for some derived formulae are

(1) $[\neg \varphi]:=[\varphi \rightarrow 0]=1-[\varphi]$;

(2) $[\varphi \vee \xi]:=[\neg(\neg \varphi \wedge \neg \xi)]=\max ([\varphi],[\xi])$;

(3) $[\varphi \leftrightarrow \xi]:=[(\varphi \rightarrow \xi) \wedge(\xi \rightarrow \varphi)]$

(4) $[\varphi \wedge \xi]:=[\neg(\varphi \rightarrow \neg \xi)]=\max (0,[\varphi]+[\xi]-1)$;

(5) $[\varphi \vee \xi]:=[\neg \varphi \rightarrow \xi]=\min (1,[\varphi]+[\xi])$;

(6) $[\exists x \varphi(x)]:=[\neg \forall x \neg \varphi(x)]:=\sup _{x \in X}[\varphi(x)]$;

(7) If $\tilde{A}, \tilde{B} \in \Im(X)$, then

(a) $[\tilde{A} \subseteq \tilde{B}]:=[\forall x(x \in \tilde{A} \rightarrow x \in \tilde{B})]=\inf _{x \in X} \min (1,1-\tilde{A}(x)+\tilde{B}(x))$;

(b) $[\tilde{A} \equiv \tilde{B}]:=[\tilde{A} \subseteq \tilde{B}] \wedge[\tilde{B} \subseteq \tilde{A}]$;

(c) $[\tilde{A} \equiv \tilde{B}]$, where $\Im(X)$ is the family of all fuzzy sets in $X$.

Definition 1 ([10]). Let $X$ be a universe of discourse, $\mu \in \Im(P(X))$ satisfying the following conditions:

(1) $\mu(x)=1, \mu(\emptyset)=1$;

(2) for any $\left\{A_{\lambda}: \lambda \in \Delta\right\}, \mu\left(\underset{\lambda \in \Delta}{\cup} A_{\lambda}\right) \geq \underset{\lambda \in \Delta}{\wedge} \mu\left(A_{\lambda}\right)$. Then $\mu$ is called a fuzzifying generalized topology and $(X, \mu)$ is a fuzzifying generalized topological space.

Definition 2 ([10]). The family of all fuzzifying generalized closed sets, denoted by $F \in \Im(P(X))$, is defined $A \in F:=X-A \in \mu$, where $X-A$ is the complement of $A$.

Definition 3 ([10]). The fuzzifying generalized neighborhood system of a point $x \in X$ is denoted by $N_{x} \in \Im(P(X))$ and defined as follows:

$N_{x}(A)=\sup _{x \in B \subseteq A} \mu(B)$.

Definition $4([10])$. The generalized closure $c_{\mu}(A)$ of $A$ is defined as $c_{\mu}(A)(x)=$ $1-N_{x}(X-A)$.

Definition 5 ([10]). For any $A \subseteq X$ the fuzzy set of the generalized interior points of $A$ is called the interior of $A$, and given as follows: $i_{\mu}(A)(x):=$ $N_{x}(A)$. Clearly, the definitions of $N_{x}(A)$ and $i_{\mu}(A)$ we have $\mu(A)=\inf _{x \in A} i_{\mu}(A)(x)$. 


\section{FUZZIFYING $\mu$-SEPARATION AXIOMS}

Remark 1. For simplicity we put the following notations:

$$
\begin{aligned}
K_{x, y}^{\mu} & :=\exists A\left(\left(A \in N_{x}^{\mu} \wedge y \notin A\right) \vee\left(A \in N_{y}^{\mu} \wedge x \notin A\right)\right), \\
H_{x, y}^{\mu} & :=\exists B \exists C\left(\left(B \in N_{x}^{\mu} \wedge y \notin B\right) \vee\left(C \in N_{y}^{\mu} \wedge x \notin C\right)\right), \\
M_{x, y}^{\mu} & :=\exists B \exists C\left(B \in N_{x}^{\mu} \wedge C \in N_{y}^{\mu} \wedge B \cap C=\emptyset\right), \\
V_{x, D}^{\mu} & :=\exists A \exists B\left(A \in N_{x}^{\mu} \wedge B \in \mu \wedge D \subseteq B \wedge A \cap B=\emptyset\right), \\
W_{A, B}^{\mu} & :=\exists G \exists H(G \in \mu \wedge H \in \mu \wedge A \subseteq G \wedge B \subseteq H \wedge G \cap H=\emptyset) .
\end{aligned}
$$

Definition 6. Let $\Omega$ be the class of all fuzzifying generalized topological spaces. The unary fuzzy predicates $\mu-T_{i} \in \mathcal{I}(\Omega), \mathrm{i}=0,1,2,3,4$ and $\mu-R_{i} \in$ $\mathcal{I}(\Omega), \mathrm{i}=0,1$ are defined as follows:

$$
\begin{aligned}
& (X, \mu) \in \mu-T_{0}:=\forall x \forall y(x \in X \wedge y \in X \wedge x \neq y) \rightarrow K_{x, y}^{\mu}, \\
& (X, \mu) \in \mu-T_{1}:=\forall x \forall y(x \in X \wedge y \in X \wedge x \neq y) \rightarrow H_{x, y}^{\mu}, \\
& (X, \mu) \in \mu-T_{2}:=\forall x \forall y(x \in X \wedge y \in X \wedge x \neq y) \rightarrow M_{x, y}^{\mu}, \\
& (X, \mu) \in \mu-T_{3}:=\forall x \forall D\left(x \in X \wedge D \in F_{i} \wedge x \notin D\right) \rightarrow V_{x, D}^{\mu}, \\
& (X, \mu) \in \mu-T_{4}:=\forall A \forall B\left(A \in F_{i} \wedge B \in F_{i} \wedge A \cap B=\emptyset\right) \rightarrow W_{A, B}^{\mu}, \\
& (X, \mu) \in \mu-R_{0}:=\forall x \forall y(x \in X \wedge y \in Y \wedge x \neq y) \rightarrow\left(K_{x, y}^{\mu} \rightarrow H_{x, y}^{\mu}\right), \\
& (X, \mu) \in \mu-R_{1}:=\forall x \forall y(x \in X \wedge y \in Y \wedge x \neq y) \rightarrow\left(K_{x, y}^{\mu} \rightarrow M_{x, y}^{\mu}\right) .
\end{aligned}
$$

\section{Lemma 1.}

(1) $\vDash M_{x, y}^{\mu} \rightarrow H_{x, y}^{\mu}$,

(2) $\vDash H_{x, y}^{\mu} \rightarrow K_{x, y}^{\mu}$,

(3) $\vDash M_{x, y}^{\mu} \rightarrow K_{x, y}^{\mu}$.

Proof.

(1) Since $\{B, c\} \in P(X): B \cap C=\emptyset\} \subseteq$ $\{B, c\} \in P(X): y \notin B \wedge x \notin C\}$, then $M_{x, y}^{\mu}=\sup _{B \cap C=\emptyset} \min \left(N_{x}^{\mu}(B)\right.$, $N_{y}^{\mu}(C) \leq \sup _{y \notin B, x \notin C} \min \left(N_{x}^{\mu}(B), N_{y}^{\mu}(C)\right)=H_{x, y}^{\mu}$.

(2) $\left[K_{x, y}^{\mu}\right]=\max \left(\sup _{y \notin A} N_{x}^{\mu}(A), \sup _{x \notin B} N_{y}^{\mu}(A)\right) \geq \sup _{y \notin A} N_{x}^{\mu}(A) \geq$ $\sup \left(N_{x}^{\mu}(A) \wedge N_{x}^{\mu}(b)=H_{x, y}^{\mu}\right.$. $y \notin A, x \notin B$

(3) From (1) and (2), it is obvious.

\section{Theorem 1.}

$(1) \vDash(X, \mu) \in \mu-T_{1} \rightarrow(X, \mu) \in \mu-T_{0}$,

$(2) \vDash(X, \mu) \in \mu-T_{2} \rightarrow(X, \mu) \in \mu-T_{1}$. 
Proof. The proof of (1) and (2) are obtained from Lemma 1 (2) and (1), respectively.

Corollary 1. $\vDash(X, \mu) \in \mu-T_{2} \rightarrow(X, \mu) \in \mu-T_{0}$.

Theorem 2. $\vDash(X, \mu) \in \mu-T_{0} \leftrightarrow(\forall x \forall y(x \in X \wedge y \in X \wedge x \neq y \rightarrow(\neg(x \in$ $\left.\left.\left.\left.c_{\mu}(\{y\})\right)\right) \vee \neg\left(y \in c_{\mu}(\{x\})\right)\right)\right)$.

Proof.

$$
\begin{aligned}
{\left[(X, \mu) \in \mu-T_{0}\right]=} & \inf _{x \neq y} \max \left(\sup _{y \notin A} N_{x}^{\mu}(A), \sup _{x \notin A} N_{y}^{\mu}(A)\right) \\
= & \inf _{x \neq y} \max \left(N_{x}^{\mu}(X \sim\{y\}), N_{y}^{\mu}(X \sim\{x\})\right) \\
= & \inf _{x \neq y} \max \left(1-c_{\mu}(\{y\})(x), 1-c_{\mu}(\{x\})(y)\right) \\
= & \inf _{x \neq y}\left(\neg\left(c_{\mu}(\{y\})(x)\right) \vee \neg\left(c_{\mu}(\{x\})(y)\right)\right) \\
= & (\forall x \forall y(x \in X \wedge y \in X \wedge x \neq y \rightarrow \\
& \left.\left.\left(\neg\left(x \in c_{\mu}(\{y\})\right)\right) \neg\left(y \in c_{\mu}(\{x\})\right)\right)\right) .
\end{aligned}
$$

Theorem 3. For any fuzzifying generalized topological space $(X, \mu), \vDash(X, \mu) \in$ $\mu-T_{1} \leftrightarrow \forall x\left(\{x\} \in F_{\mu}\right)$.

Proof. For any $x_{1}, x_{2}, x_{1} \neq x_{2}$,

$$
\begin{aligned}
{\left[\forall x\left(\{x\} \in F_{\mu}\right)\right] } & =\inf _{x \in X} F_{\mu}(\{x\}) \\
& =\inf _{x \in X} \mu(X \sim\{x\}) \\
& =\inf _{x \in X} \inf _{y \in X \sim\{x\}} N_{y}^{\mu}(X \sim\{x\}) \\
& \leq \inf _{y \in X \sim\left\{x_{2}\right\}} N_{y}^{\mu}\left(X \sim\left\{x_{2}\right\}\right) \\
& \leq N_{x_{1}}^{\mu}\left(X \sim\left\{x_{2}\right\}\right)=\sup _{x_{2} \notin A} N_{x_{1}}^{\mu}(A) .
\end{aligned}
$$

Similarly, we have, $\left[\forall x\left(\{x\} \in F_{\mu}\right)\right] \leq \sup _{x_{1} \notin B} N_{x_{2}}^{\mu}(B)$. Then,

$$
\begin{aligned}
{\left[\forall x\left(\{x\} \in F_{\mu}\right)\right] } & =\inf _{x_{1} \neq x_{2}} \min \left(\sup _{x_{2} \notin A} N_{x_{1}}^{\mu}(A), \sup _{x_{1} \in B} N_{x_{2}}^{\mu}(B)\right) \\
& =\inf _{x_{1} \neq x_{2}} \sup _{x_{1} \notin B, x_{2} \notin A} \min \left(N_{x_{1}}^{\mu}(A), N_{x_{2}}^{\mu}(B)\right) \\
& =\left[(X, \mu) \in \mu-T_{1}\right] .
\end{aligned}
$$


On the other hand,

$$
\begin{aligned}
{\left[(X, \mu) \in \mu-T_{1}\right] } & =\inf _{x_{1} \neq x_{2}} \min \left(\sup _{x_{2} \notin A} N_{x_{1}}^{\mu}(A), \sup _{x_{1} \in B} N_{x_{2}}^{\mu}(B)\right) \\
& =\inf _{x_{1} \neq x_{2}} \min \left(N_{x_{1}}^{\mu}\left(X \sim\left\{x_{2}\right\}\right), N_{x_{2}}^{\mu}\left(X \sim\left\{x_{1}\right\}\right)\right) \\
& \leq \inf _{x_{1} \neq x_{2}} N_{x_{1}}^{\mu}\left(X \sim\left\{x_{2}\right\}\right) \\
& =\inf _{x_{2} \in X} \inf _{x_{1} \in X \sim\left\{x_{2}\right\}} N_{x_{1}}^{\mu}\left(X \sim\left\{x_{2}\right\}\right) \\
& =\inf _{x_{2} \in X} \mu\left(X \sim\left\{x_{2}\right\}\right) \\
& =\inf _{x \in X} \mu(X \sim\{x\}) \\
& =\left[\forall x\left(\{x\} \in F_{\mu}\right)\right] .
\end{aligned}
$$

Thus, $\left[(X, \mu) \in \mu-T_{1}\right]=\left[\forall x\left(\{x\} \in F_{\mu}\right)\right]$.

Definition 7. The $\mu$-local base $\mathcal{S} \beta_{x}$ of $x$ is a function from $P(X)$ into $I$ such that the following conditions are satisfied:

(1) $\vDash \mathcal{S} \beta_{x} \subseteq N_{x}^{\mu}$,

(2) $\vDash A \in N_{x}^{\mu} \rightarrow \exists B\left(B \in \mathcal{S} \beta_{x} \wedge x \in B \subseteq A\right)$.

Lemma 2. $\vDash A \in N_{x}^{\mu} \leftrightarrow \exists B\left(B \in \mathcal{S} \beta_{x} \wedge x \in B \subseteq A\right)$.

Proof. From condition (1) in Definition 7 we have $N_{x}^{\mu}(A) \geq N_{x}^{\mu}(B) \geq$ $\mathcal{S} \beta_{x}(B)$ for each $B \in P(X)$ such that $x \in B \subseteq A$. So, $N_{x}^{\mu}(A) \geq \sup _{x \in B \subseteq A} \mathcal{S} \beta_{x}(B)$. From condition (2) in Definition $7, N_{x}^{\mu}(A) \leq \sup _{x \in B \subseteq A} \mathcal{S} \beta_{x}(B)$. Hence $N_{x}^{\mu}(A)=$ $\sup _{x \in B \subseteq A} \mathcal{S} \beta_{x}(B)$.

Theorem 4. If $\mathcal{S} \beta_{x}$ is a $\mu$-local basis of $x$, then $\vDash(X, \mu) \in \mu-T_{2} \leftrightarrow$ $\forall x \forall y\left(x \in X \wedge y \in X \wedge x \neq y \rightarrow \exists B\left(B \in \mathcal{S} \beta_{x} \wedge y \in \neg\left(c_{\mu}(B)\right)\right)\right)$.

Proof. $\left[\forall x \forall y\left(x \in X \wedge y \in X \wedge x \neq y \rightarrow \exists B\left(B \in \mathcal{S} \beta_{x} \wedge y \in \neg\left(c_{\mu}(B)\right)\right)\right)\right]$

$$
\begin{aligned}
= & \inf _{x \neq y} \sup _{B \in P(X)} \min \left(\mathcal{S} \beta_{x}(B), N_{y}^{\mu}(X \sim B)\right) \\
= & \inf _{x \neq y} \sup _{B \in P(X)} \sup _{y \in C \subseteq X \sim B} \min \left(\mathcal{S} \beta_{x}(B), \mathcal{S} \beta_{y}(C)\right) \\
= & \inf _{x \neq y} \sup _{B \cap C=\emptyset \in D \subseteq B} \sup _{x \in D \subseteq E} \min \left(\mathcal{S} \beta_{x}(D), \mathcal{S} \beta_{y}(E)\right) \\
= & \inf _{x \neq y} \sup _{B \cap C=\emptyset} \min \left(\sup _{x \in D \subseteq B} \mathcal{S} \beta_{x}(D), \sup _{y \in E \subseteq C} \mathcal{S} \beta_{y}(E)\right) \\
= & \inf _{x \neq y} \sup _{B \cap C=\emptyset} \min \left(N_{x}^{\mu}(B), N_{y}^{\mu}(C)\right) \\
= & {\left[(X, \mu) \in \mu-T_{2}\right] . }
\end{aligned}
$$


Theorem 5. $\vDash(X, \mu) \in \mu-R_{1} \rightarrow(X, \mu) \in \mu-R_{0}$.

Proof. From Lemma 1 (1) the proof is immediate.

\section{Theorem 6.}

(1) $\vDash(X, \mu) \in \mu-T_{1} \rightarrow(X, \mu) \in \mu-R_{0}$,

(2) $\vDash(X, \mu) \in \mu-T_{1} \rightarrow(X, \mu) \in \mu-R_{0} \wedge(X, \mu) \in \mu-T_{0}$,

(3) If $\mu-T_{0}(X, \mu)=1$, then

$$
\vDash(X, \mu) \in \mu-T_{1} \leftrightarrow(X, \mu) \in \mu-R_{0} \wedge(X, \mu) \in \mu-T_{0} .
$$

Proof.

(1) We have,

$$
\mu-R_{1}(X, \mu)=\inf _{x \neq y}\left[H_{x, y}^{\mu}\right] \leq \inf _{x \neq y}\left[K_{x, y}^{\mu} \rightarrow H_{x, y}^{\mu}\right]=\mu-R_{0}(X, \mu) .
$$

(2) It is obtained from (1) and from Theorem 1(1).

(3) Since $\mu-T_{0}(X, \mu)=1$, for every $x, y \in X$ such that $x \neq y$ we have $\left[K_{x, y}^{\mu}\right]=1$. Now

$$
\begin{aligned}
{\left[(X, \mu) \in \mu-R_{0} \wedge(X, \mu) \in \mu-T_{0}\right] } & =\left[(X, \mu) \in-\mu-R_{0}\right] \\
& =\inf _{x \neq y} \min \left(1,1-\left[K_{x, y}^{\mu}\right]+\left[H_{x, y}^{\mu}\right]\right) \\
& =\inf _{x \neq y}\left[H_{x, y}^{\mu}\right]=-\mu-T_{1}(X, \mu) .
\end{aligned}
$$

\section{Theorem 7 .}

(1) $\vDash(X, \mu) \in \mu-R_{0} \wedge(X, \mu) \in \mu-T_{0} \rightarrow(X, \mu) \in \mu-T_{1}$;

(2) If $\mu-T_{0}(X, \mu)=1$, then

$$
\vDash(X, \mu) \in \mu-R_{0} \wedge(X, \mu) \in \mu-T_{0} \leftrightarrow(X, \mu) \in \mu-T_{1} .
$$

Proof.

(1) $\left[(X, \mu) \in \mu-R_{0} \wedge(X, \mu) \in \mu-T_{0}\right]$

$$
\begin{aligned}
& =\max \left(0,-\mu-R_{0}(X, \mu)+-\mu-T_{0}(X, \mu)-1\right) \\
& =\max \left(0, \inf _{x \neq y} \min \left(1,1-\left[K_{x, y}^{\mu}\right]+\left[H_{x, y}^{\mu}\right]\right)+\inf _{x \neq y}\left[K_{x, y}^{\mu}\right]-1\right) \\
& \leq \max \left(0, \inf _{x \neq y} \min \left(1,1-\left[K_{x, y}^{\mu}\right]+\left[H_{x, y}^{\mu}\right]\right)+\left[K_{x, y}^{\mu}\right]-1\right) \\
& =\inf _{x \neq y}\left[H_{x, y}^{\mu}\right]=-\mu-T_{1}(X, \mu) .
\end{aligned}
$$

(2) $\left[(X, \mu) \in \mu-R_{0} \wedge(X, \mu) \in \mu-T_{0}\right]$

$$
\begin{aligned}
& =\left[(X, \mu) \in-\mu-R_{0}\right]=\inf _{x \neq y} \min \left(1,1-\left[K_{x, y}^{\mu}\right]+\left[H_{x, y}^{\mu}\right]\right) \\
& =\inf _{x \neq y}\left[H_{x, y}^{\mu}\right]=-\mu-T_{1}(X, \mu),
\end{aligned}
$$


because $\mu-T_{0}(X, \mu)=1$, we have for each $x, y \in X$ such that $x \neq y,\left[K_{x, y}^{\mu}\right]=1$.

\section{Theorem 8.}

$(1) \vDash(X, \mu) \in \mu-T_{0} \rightarrow\left((X, \mu) \in \mu-R_{0} \rightarrow(X, \mu) \in \mu-T_{1}\right)$.

(2) $\vDash(X, \mu) \in \mu-R_{0} \rightarrow\left((X, \mu) \in \mu-T_{0} \rightarrow(X, \mu) \in \mu-T_{1}\right)$.

Proof. From Theorems 6 (1) and 7 (1), we have

(1) $\left[(X, \mu) \in \mu-T_{0} \rightarrow\left((X, \mu) \in \mu-R_{0} \rightarrow(X, \mu) \in \mu-T_{1}\right)\right]$

$$
\begin{aligned}
= & \min \left(1,1-\left[(X, \mu) \in-\mu-T_{0}\right]\right. \\
& \left.+\min \left(1,1-\left[(X, \mu) \in-\mu-R_{0}\right]+\left[(X, \mu) \in-\mu-T_{1}\right]\right)\right) \\
= & \min \left(1,1-\left[(X, \mu) \in-\mu-T_{0}\right]\right. \\
& \left.+1-\left[(X, \mu) \in-\mu-R_{0}\right]+\left[(X, \mu) \in-\mu-T_{1}\right]\right) \\
= & \min \left(1,1-\left(\left[(X, \mu) \in-\mu-T_{0}\right]\right.\right. \\
& \left.\left.+\left[(X, \mu) \in-\mu-R_{0}\right]-1\right)+\left[(X, \mu) \in-\mu-T_{1}\right]\right)=1 .
\end{aligned}
$$

(2) $\left[(X, \mu) \in \mu-R_{0} \rightarrow\left((X, \mu) \in \mu-T_{0} \rightarrow(X, \mu) \in \mu-T_{1}\right)\right]$.

$$
\begin{aligned}
= & \min \left(1,1-\left(\left[(X, \mu) \in-\mu-T_{0}\right]\right.\right. \\
& \left.\left.+\left[(X, \mu) \in-\mu-R_{0}\right]-1\right)+\left[(X, \mu) \in-\mu-T_{1}\right]\right)=1 .
\end{aligned}
$$

\section{Theorem 9.}

$(1) \vDash(X, \mu) \in \mu-T_{2} \rightarrow(X, \mu) \in \mu-R_{1}$,

$(2) \vDash(X, \mu) \in \mu-T_{2} \rightarrow(X, \mu) \in \mu-R_{1} \wedge(X, \mu) \in \mu-T_{0}$;

(3) If $\mu-T_{0}(X, \mu)=1$, then $\vDash(X, \mu) \in \mu-T_{2} \leftrightarrow(X, \mu) \in \mu-R_{1} \wedge(X, \mu) \in \mu-T_{0}$.

Proof.

(1) We have $\mu-T_{2}(X, \mu)=\inf _{x \neq y}\left[M_{x, y}^{\mu}\right] \leq \inf _{x \neq y}\left[K_{x, y}^{\mu} \rightarrow M_{x, y}^{\mu}\right]=\mu-R_{1}(X, \mu)$.

(2) It is obtained from (1) and Corollary 1.

(3) Since $\mu-T_{0}(X, \mu)=1$, then for each $x, y \in X$ such that $x \neq y$ we have $\left[K_{x, y}^{\mu}\right]=1$.

Now,

$$
\begin{aligned}
& {\left[(X, \mu) \in \mu-R_{1} \wedge(X, \mu) \in \mu-T_{0}\right] } \\
= & {\left[(X, \mu) \in-\mu-R_{1}\right]=\inf _{x \neq y} \min \left(1,1-\left[K_{x, y}^{\mu}\right]+\left[M_{x, y}^{\mu}\right]\right) } \\
= & \inf _{x \neq y}\left[M_{x, y}^{\mu}\right]=-\mu-T_{2}(X, \mu) .
\end{aligned}
$$

\section{Theorem 10.}

$(1) \vDash(X, \mu) \in \mu-R_{1} \wedge(X, \mu) \in \mu-T_{0} \rightarrow(X, \mu) \in \mu-T_{2}$.

(2) $\vDash(X, \mu) \in \mu-R_{1} \wedge(X, \mu) \in \mu-T_{0} \leftrightarrow(X, \mu) \in \mu-T_{2}$. 
Proof.

(1) $\left[(X, \mu) \in \mu-R_{1} \wedge(X, \mu) \in \mu-T_{0}\right]$

$$
\begin{aligned}
& =\max \left(0,-\mu-R_{1}(X, \mu)+-\mu-T_{0}(X, \mu)-1\right) \\
& =\max \left(0, \inf _{x \neq y} \min \left(1,1-\left[K_{x, y}^{\mu}\right]+\left[M_{x, y}^{\mu}\right]\right)+\inf _{x \neq y}\left[K_{x, y}^{\mu}\right]-1\right) \\
& \leq \max \left(0, \inf _{x \neq y} \min \left(1,1-\left[K_{x, y}^{\mu}\right]+\left[M_{x, y}^{\mu}\right]\right)+\left[K_{x, y}^{\mu}\right]-1\right) \\
& =\inf _{x \neq y}\left[M_{x, y}^{\mu}\right]=-\mu-T_{2}(X, \mu) .
\end{aligned}
$$

(2) $\left[(X, \mu) \in \mu-R_{1} \wedge(X, \mu) \in \mu-T_{0}\right]$

$$
\begin{aligned}
& =\left[(X, \mu) \in-\mu-R_{1}\right]=\inf _{x \neq y} \min \left(1,1-\left[K_{x, y}^{\mu}\right]+\left[M_{x, y}^{\mu}\right]\right) \\
& =\inf _{x \neq y}\left[M_{x, y}^{\mu}\right]=-\mu-T_{2}(X, \mu),
\end{aligned}
$$

since $\mu-T_{0}(X, \mu)=1$, then for each $x, y \in X$ such that $x \neq y,\left[K_{x, y}^{\mu}\right]=1$.

\section{Theorem 11.}

(1) $\vDash(X, \mu) \in \mu-T_{0} \rightarrow\left((X, \mu) \in \mu-R_{1} \rightarrow(X, \mu) \in \mu-T_{2}\right)$.

$(2) \vDash(X, \mu) \in \mu-R_{1} \rightarrow\left((X, \mu) \in \mu-T_{0} \rightarrow(X, \mu) \in \mu-T_{2}\right)$.

Proof.

(1) From Theorems 9(1) and 10(1) we have

$$
\begin{aligned}
& {\left[(X, \mu) \in \mu-T_{0} \rightarrow\left((X, \mu) \in \mu-R_{1} \rightarrow(X, \mu) \in \mu-T_{2}\right)\right] } \\
= & \min \left(1,1-\left[(X, \mu) \in-\mu-T_{0}\right]+\min \left(1,1-\left[(X, \mu) \in-\mu-R_{1}\right]+\left[(X, \mu) \in-\mu-T_{2}\right]\right)\right) \\
= & \min \left(1,1-\left[(X, \mu) \in-\mu-T_{0}\right]+1-\left[(X, \mu) \in-\mu-R_{1}\right]+\left[(X, \mu) \in-\mu-T_{2}\right]\right) \\
= & \min \left(1,1-\left(\left[(X, \mu) \in-\mu-T_{0}\right]+\left[(X, \mu) \in-\mu-R_{1}\right]-1\right)+\left[(X, \mu) \in-\mu-T_{2}\right]\right)=1 .
\end{aligned}
$$

(2) The proof is similar to (1).

Theorem 12. If $\mu-T_{0}(X, \mu)=1$, then

$$
\begin{aligned}
(1) & \vDash\left((X, \mu) \in \mu-T_{0} \rightarrow\left((X, \mu) \in \mu-R_{0} \rightarrow(X, \mu) \in \mu-T_{1}\right)\right) \\
& \wedge\left((X, \mu) \in \mu-T_{1} \rightarrow \neg\left((X, \mu) \in \mu-T_{0} \rightarrow \neg\left((X, \mu) \in \mu-R_{0}\right)\right)\right), \\
(2) & \vDash\left((X, \mu) \in \mu-R_{0} \rightarrow\left((X, \mu) \in \mu-T_{0} \rightarrow(X, \mu) \in \mu-T_{1}\right)\right) \\
& \wedge\left((X, \mu) \in \mu-T_{1} \rightarrow \neg\left((X, \mu) \in \mu-T_{0} \rightarrow \neg\left((X, \mu) \in \mu-R_{0}\right)\right)\right), \\
(3) & \vDash\left((X, \mu) \in \mu-T_{0} \rightarrow\left((X, \mu) \in \mu-R_{0} \rightarrow(X, \mu) \in \mu-T_{1}\right)\right) \\
& \wedge\left((X, \mu) \in \mu-T_{1} \rightarrow \neg\left((X, \mu) \in \mu-R_{0} \rightarrow \neg\left((X, \mu) \in \mu-T_{0}\right)\right)\right), \\
(4) & \vDash\left((X, \mu) \in \mu-R_{0} \rightarrow\left((X, \mu) \in \mu-T_{0} \rightarrow(X, \mu) \in \mu-T_{1}\right)\right) \\
& \wedge\left((X, \mu) \in \mu-T_{1} \rightarrow \neg\left((X, \mu) \in \mu-R_{0} \rightarrow \neg\left((X, \mu) \in \mu-T_{0}\right)\right)\right) .
\end{aligned}
$$

Proof. For simplicity we put, $\mu-T_{0}(X, \mu)=\lambda, \mu-R_{0}(X, \mu)=\theta$ and $\mu-T_{1}(X, \mu)=$ $\rho$. Now, applying Theorem $7(2)$, the proof is obtained with some relations 
in fuzzy logic as follows:

$$
\begin{aligned}
(1) & =(\lambda \underset{\circ}{\wedge} \theta \leftrightarrow \rho)=(\lambda \underset{\circ}{\wedge} \theta \rightarrow \rho) \wedge(\rho \rightarrow \lambda \underset{\circ}{\wedge} \theta) \\
& =(\lambda \underset{\circ}{\wedge} \theta \wedge \neg \rho) \wedge \neg(\rho \wedge \neg(\lambda \wedge \underset{\circ}{\wedge} \theta)) \\
& =\neg(\lambda \underset{\circ}{\wedge} \neg(\neg(\theta \wedge \neg \rho))) \wedge \neg\left(\rho \wedge_{\circ}(\lambda \rightarrow \neg \theta)\right) \\
& =(\lambda \rightarrow \neg(\theta \wedge \neg \rho)) \wedge(\rho \rightarrow \neg(\lambda \rightarrow \neg \theta)) \\
& =(\lambda \rightarrow(\theta \rightarrow \rho)) \wedge(\rho \rightarrow \neg(\lambda \rightarrow \neg \theta)),
\end{aligned}
$$

since $\hat{\sim}_{0}$ is commutative one can have the proof of statements $(2)-(4)$ in a similar way as (1).

By a similar procedure to Theorem 11 one can have the following theorem.

\section{Theorem 13.}

$(1) \vDash\left((X, \mu) \in \mu-T_{0} \rightarrow\left((X, \mu) \in \mu-R_{1} \rightarrow(X, \mu) \in \mu-T_{2}\right)\right)$ $\wedge\left((X, \mu) \in \mu-T_{2} \rightarrow \neg\left((X, \mu) \in \mu-T_{0} \rightarrow \neg\left((X, \mu) \in \mu-R_{1}\right)\right)\right)$,

$(2) \vDash\left((X, \mu) \in \mu-R_{1} \rightarrow\left((X, \mu) \in \mu-T_{0} \rightarrow(X, \mu) \in \mu-T_{2}\right)\right)$ $\wedge\left((X, \mu) \in \mu-T_{2} \rightarrow \neg\left((X, \mu) \in \mu-T_{0} \rightarrow \neg\left((X, \mu) \in \mu-R_{1}\right)\right)\right)$,

$(3) \vDash\left((X, \mu) \in \mu-T_{0} \rightarrow\left((X, \mu) \in \mu-R_{1} \rightarrow(X, \mu) \in \mu-T_{2}\right)\right)$ $\wedge\left((X, \mu) \in \mu-T_{2} \rightarrow \neg\left((X, \mu) \in \mu-R_{1} \rightarrow \neg\left((X, \mu) \in \mu-T_{0}\right)\right)\right)$,

$(4) \vDash\left((X, \mu) \in \mu-R_{1} \rightarrow\left((X, \mu) \in \mu-T_{0} \rightarrow(X, \mu) \in \mu-T_{2}\right)\right)$ $\wedge\left((X, \mu) \in \mu-T_{2} \rightarrow \neg\left((X, \mu) \in \mu-R_{1} \rightarrow \neg\left((X, \mu) \in \mu-T_{0}\right)\right)\right)$.

\section{Lemma 3.}

(1) If $D \subseteq B$, then $\sup _{A \cap B=\emptyset} N_{x}^{\mu}(A)=\sup _{A \cap B=\emptyset, D \subseteq B} N_{x}^{\mu}(Z)$;

(2) $\sup _{A \cap B=\emptyset} \inf _{y \in D} N_{y}^{\mu}(X \sim A)=\sup _{\substack{A \cap B=\emptyset \\ D \subseteq B}} \mu(B)$.

Proof.

(1) Since $D \subseteq B$,

$$
\sup _{A \cap B=\emptyset} N_{x}^{\mu}(A)=\sup _{A \cap B=\emptyset} N_{x}^{\mu}(A) \wedge[D \subseteq B]=\sup _{\substack{A \cap B=\emptyset \\ D \subseteq B}} N_{x}^{\mu}(A) .
$$

(2) Let $y \in D$ and $A \cap B=\emptyset$. Then,

$$
\begin{aligned}
\sup _{\substack{A \cap B=\bar{D} \\
D \subseteq B}} \mu(B) & =\sup _{\substack{A \cap B=\emptyset \\
D \subseteq B}} \mu(B) \wedge[y \in D] \\
& =\sup _{y \in D \subseteq B \subseteq X \sim A} \mu(B) \\
& =\sup _{y \in D \subseteq X \sim A} \mu(B) \\
& =\sup _{A \cap B=\emptyset} \inf _{y \in D} N_{y}^{\mu}(X \sim A) .
\end{aligned}
$$


Definition 8. $\mu-T_{3}^{(1)}(X, \mu):=$

$$
\forall x \forall D\left(x \in X \wedge D \in F_{i} \wedge x \notin D \rightarrow \exists A\left(A \in N_{x}^{\mu} \wedge\left(D \subseteq X \sim c_{\mu}(A)\right)\right)\right) .
$$

Theorem 14. $\vDash(X, \mu) \in \mu-T_{3} \leftrightarrow(X, 1,2) \in \mu-T_{3}^{(1)}$.

Proof. Now,

$$
\begin{aligned}
\mu-T_{3}^{(1)}(X, \mu)= & \inf _{x \notin D} \min (1,1-i(X \sim D) \\
& \left.+\sup _{A \in P(X)} \min \left(N_{x}^{\mu}(A), \inf _{y \in D}\left(1-c_{\mu}(A)(y)\right)\right)\right), \\
= & \inf _{x \notin D} \min (1,1-i(X \sim D) \\
& \left.+\sup _{A \in P(X)} \min \left(N_{x}^{\mu}(A), \inf _{y \in D} N_{y}^{\mu}(X \sim A)\right)\right),
\end{aligned}
$$

and

$$
\mu-T_{3}(X, \mu)=\inf _{x \notin D} \min \left(1,1-i(X \sim D)+\sup _{\substack{A \cap B=\emptyset \\ D \subseteq \bar{B}}} \min \left(N_{x}^{\mu}(A), \mu(B)\right)\right) .
$$

So the result hold if we prove that

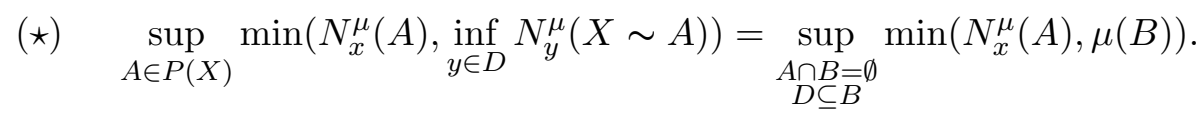

It is clear that, on the left hand side of $(\star)$ when $A \cap C \neq \emptyset$, then there exists $y \in X$ such that $y \in D$ and $y \notin X \sim A$. So $\inf _{y \in D} N_{y}^{\mu}(X \sim A)=0$ and thus $(\star)$ becomes

$$
\sup _{\substack{A \in P(X) \\ A \cap B=\emptyset}} \min \left(N_{x}^{\mu}(A), \inf _{y \in D} N_{y}^{\mu}(X \sim A)\right)=\sup _{\substack{A \cap B=\emptyset \\ D \subseteq B}} \min \left(N_{x}^{\mu}(A), \mu(B)\right),
$$

which is obtained from Lemma 3 .

Definition 9. $\mu-T_{3}^{(2)}(X, \mu):=\forall x \forall B(x \in B \wedge B \in \mu \rightarrow$

$$
\left.\exists A\left(A \in N_{x}^{\mu} \wedge c_{\mu}(A) \subseteq B\right)\right) .
$$

Theorem 15. $\vDash(X, \mu) \in \mu-T_{3} \leftrightarrow(X, \mu) \in \mu-T_{3}^{(2)}$.

Proof. From Theorem 14, we have $\mu-T_{3}(X, \mu)$

$=\inf _{x \notin D} \min \left(1,1-\mu(X \sim D)+\sup _{A \in P(X)} \min \left(N_{x}^{\mu}(A), \inf _{y \in D} N_{y}^{\mu}(X \sim A)\right)\right)$. 
Now, if we put $B=X \sim D$, then

$$
\begin{aligned}
& \mu-T_{3}^{(2)}(X, \mu) \\
= & \inf _{x \in B} \min \left(1,1-i(B)+\sup _{A \in P(X)} \min \left(N_{x}^{\mu}(A), \inf _{y \in X \sim B} N_{y}^{\mu}(X \sim A)\right)\right) \\
= & \inf _{x \notin D} \min \left(1,1-\mu(X \sim D)+\sup _{A \in P(X)} \min \left(N_{x}^{\mu}(A), \inf _{y \in D} N_{y}^{\mu}(X \sim A)\right)\right) \\
= & \mu-T_{3}(X, \mu) .
\end{aligned}
$$

Acknowledgment. The authors are thankful to the referee for some constructive comments and suggestions towards some improvements of the earlier version of the paper.

\section{REFERENCES}

[1] U. Hohle, Many Valued Topology and its Applications. Kluwer Academic Publishers, Dordrecht (2001).

[2] U. Hohle and S. E. Rodabaugh, Mathematics of Fuzzy Sets: Logic, Topology, and Measure Theory in Handbook of Fuzzy Sets Series. Kluwer Academic Publishers, Dordrecht. 3(1999).

[3] U. Hohle, S. E. Rodabaugh and A. Sostak, Special Issue on Fuzzy Topology. Fuzzy Sets and Systems, 73 (1995), 1-183.

[4] F.H. Khedr, F.M. Zeyada and O.R. Sayed, On separation axioms in fuzzifying topology, Fuzzy Sets and Systems, 119 (2001), 439-458.

[5] T. Kubiak, On Fuzzy Topologies, Ph. D. Thesis, Adam Mickiewicz University, Poznan, Poland (1985).

[6] Y. M. Liu and M. K. Luo, Fuzzy Topology. Singapore, World Scientific (1998).

[7] G. J. Wang, Theory of L-Fuzzy generalized topological Spaces. Shanxi Normal University Press (in Chinese) (1988).

[8] C. L. Chang, Fuzzy topological spaces. J. Math. Anal. Appl., 24 (1968), 182-190.

[9] J. A. Goguen, The fuzzy Tychonoff Theorem. J. Math. Anal. Appl., 43 (1973), 182190.

[10] N. Gowrisankar, N. Rajesh and V. Vijayabharathi, Fuzzifying generalized topology (under preparation).

[11] J. Shen, Separation axiom in fuzzifying topology, Fuzzy Sets and Systems 57 (1993), 111-123.

[12] O.R. Sayed, $\beta$-separartion axioms based on Lukasiewicz logic, Engineering Science Letters, 1:1 (2012), 1-24.

[13] S. Tahiliani, On study of some weak separation axioms using $\beta$-open sets and $\beta$ closure operator, Italian Journal of Pure and Applied Mathematics, 24 (2008), 67-76. 
N. Gowrisankar

70/232 6B, Kollupettai street

M. Chavady

ThanjaVuR-613001

TAMILNADU

INDIA

E-mail address: gowrisankartnj@gmail.com

N. RAJESH

Department of Mathematics

Rajah Serfoji Govt. College

ThanjaVur-613005

TAMILNADU

INDIA

E-mail address: nrajesh_topology@yahoo.co.in

\section{VIJAYABHARATHI}

Department of Mathematics

National Institute of Technology

Tiruchirappalli

TAMilnadu

INDIA

E-mail address: vijayabharathi_v@yahoo.com 